\title{
Long-term follow-up of cytogenetically normal CEBPA-mutated AML
}

\author{
Friederike Pastore ${ }^{1,2,3^{*}}$, Daniela Kling ${ }^{1}$, Eva Hoster ${ }^{1,4}$, Annika Dufour ${ }^{1}$, Nikola P Konstandin ${ }^{1}$, Stephanie Schneider ${ }^{1}$, \\ Maria C Sauerland ${ }^{5}$, Wolfgang E Berdel ${ }^{6}$, Thomas Buechner ${ }^{6}$, Bernhard Woermann ${ }^{7}$, Jan Braess ${ }^{8}$, \\ Wolfgang Hiddemann ${ }^{1,2,3}$ and Karsten Spiekermann ${ }^{1,2,3}$
}

\begin{abstract}
Background: The aim of this study was to analyze the long-term survival of AML patients with CEBPA mutations. Patients and methods: We investigated 88 AML patients with a median age of 61 years and (1) cytogenetically normal AML (CN-AML), (2) monoallelic (moCEBPA) or biallelic (biCEBPA) CEBPA mutation, and (3) intensive induction treatment. 60/88 patients have been described previously with a shorter follow-up.

Results: Median follow-up time was 9.8 years (95\% Cl: 9.4-10.1 years) compared to 3.2 and 5.2 years in our former analyses. Patients with biCEBPA mutations survived significantly longer compared to those with moCEBPA (median overall survival (OS) 9.6 years vs. 1.7 years, $p=0.008$ ). Patients $\leq 60$ years and biCEBPA mutations showed a favorable prognosis with a 10-year OS rate of $81 \%$.

Both, bi- and moCEBPA-mutated groups had a low early death (d60) rate of $7 \%$ and $9 \%$, respectively. Complete remission (CR) rates for biCEBPA- and moCEBPA-mutated patients were $82 \%$ vs. $70 \%$ ( $p=0.17$ ). biCEBPA-mutated patients showed a longer relapse free survival (RFS) (median RFS 9.4 years vs. 1.5 years, $p=0.021$ ) and a lower cumulative incidence of relapse (CIR) compared to moCEBPA-mutated patients. These differences in OS and RFS were confirmed after adjustment for known clinical and molecular prognostic factors.

Conclusions: In this long-term observation we confirmed the favorable prognostic outcome of patients with biCEBPA mutations compared to moCEBPA-mutated CN-AML. The high probability of OS (81\%) in younger patients is helpful to guide intensity of postremission therapy.
\end{abstract}

Keywords: Acute Myeloid Leukemia, Cytogenetically normal AML, Monoallelic CEBPA mutation, Biallelic CEBPA mutation

\section{Background}

According to the current recommendations of the WHO [1] the large and heterogeneous group of cytogenetically normal AML (CN-AML) is further stratified by the presence or absence of internal tandem duplications of fms-related tyrosine kinase 3 (FLT3-ITD), mutations of nucleophosmin (NPM1) and mutations in the CCAAT/ enhancer binding protein (C/EBP) alpha $(C E B P A)$. In fact, "AML with mutated CEBPA" has been classified as its own category in the current WHO classification [1].

\footnotetext{
* Correspondence: Friederike.Pastore@med.uni-muenchen.de 'Laboratory for Leukemia Diagnostics, Department of Internal Medicine III, University Hospital Munich Grosshadern, Munich, Germany ${ }^{2}$ German Cancer Consortium (DKTK), Heidelberg, Germany Full list of author information is available at the end of the article
}

The gene encoding for the CCAAT/enhancer binding protein- $\alpha$ (CEBPA) is located on chromosome 19 band q13.11. It was first full-length cloned in 1997 [2]. The CEBPA protein is $42 \mathrm{kDa}$ of size. It is expressed in myelomonocytic cells and upregulated in granulocytic differentiation acting as a myeloid transcription factor. Mutations of CEBPA in AML were first described in 2001 [3]. Nterminal frameshift mutations lead to the overexpression of a truncated $30 \mathrm{kDA}$ isoform of $C E B P A$ that suppresses $C E B P A$ function in a dominant negative way. $\mathrm{C}$-terminal mutations occur mainly in the basic Zipper (bZIP) domain of $C E B P A$, and impair its function to homodimerize and heterodimerize with other proteins as well as its DNA binding [4]. CEBPA knock-out mice show a selective block

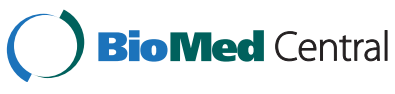

(c) 2014 Pastore et al.; licensee BioMed Central Ltd. This is an Open Access article distributed under the terms of the Creative Commons Attribution License (http://creativecommons.org/licenses/by/4.0), which permits unrestricted use, distribution, and reproduction in any medium, provided the original work is properly credited. The Creative Commons Public Domain Dedication waiver (http://creativecommons.org/publicdomain/zero/1.0/) applies to the data made available in this article, unless otherwise stated. 
of differentiation lacking mature granulocytes while other hematopoietic cells differentiate regularly [5]. Mutations of CEBPA have been shown to be associated with $\mathrm{CN}$-AML where they occur with frequencies of $8-18 \%$ [6-10] and with the French-American-British (FAB) subtypes M1 and M2 [11].

CEBPA mutations can occur as monoallelic mutations (moCEBPA) or as biallelic mutations (biCEBPA). Patients with biCEBPA mutations usually have a C-terminal mutation on one allele and an N-terminal mutation on the other allele, resulting in a lack of CEBPA wildtype allele expression [12,13]. We and others have reported earlier that the positive prognostic impact on outcome is restricted to patients with biCEBPA mutations [6,13-16]. Except for the studies of Taskesen [16] and Green [14] patient numbers with mutated $C E B P A$ at diagnosis were small $(\mathrm{n}<50)$ and median follow-up times were $<10$ years in the majority of the upper mentioned analyses.

The objective of this study was to investigate if the effect of moCEBPA versus (vs.) biCEBPA mutations on outcome was true also in a longer follow-up period and to elucidate the clinical course of disease in biCEBPA mutations patients.

\section{Methods}

\section{Patients}

In this analysis we included all cytogenetically normal (CN) AML patients with a monoallelic or a biallelic CEBPA mutation treated within the two large multicenter AML Cooperative group clinical studies, the AMLCG99 trial [NCT00266136] and the AMLCG2008 trial [NCT01382147; EUDRACT2007-003103-12] (randomization from July 1999 until December 2012; approved by the local institutional review boards) or in analogy to clinical studies (treatment start from April 2000 until March 2013) in our university hospital. We identified 88 patients fulfilling these criteria. A subset of 60 of these patients have been investigated in previous publications with a shorter followup $[6,17]$.

Clinical parameters available at first diagnosis included age, sex, Eastern Cooperative Group (ECOG) performance status [18], the French-American-British (FAB) morphologic AML subtype, the origin of AML (de novo vs. secondary or therapy-related AML), white blood cell count (WBC), platelet count, haemoglobin level, lactate dehydrogenase (LDH) level, myeloid blasts in the bone marrow (BM) and in the peripheral blood (PB).

All patients were treated with intensive induction chemotherapy. 76 patients $(86 \%)$ were treated within the AMLCG99 $(n=68)$ and AMLCG2008 $(n=7)$ and the HD98-A study $(n=1)$. Details of the AMLCG99 study and AMLCG2008 study have been published before [19-21]. 12 patients (14\%) were treated in analogy to the AMLCG studies or with a classical " $7+3$ " therapeutic regimen. The analysis included 19 patients who underwent allogeneic stem cell transplantation (SCT): as consolidation in first CR ( $\mathrm{n}=7 ; 8 \%$ of patients), at the time of relapse $(\mathrm{n}=8 ; 9.1 \%)$ or primary refractory disease $(\mathrm{n}=4 ; 4.5 \%)$. The studies were approved by the ethics committees of all participating institutions.

\section{Cytogenetic and molecular analysis}

Cytogenetic and molecular analyses were performed on BM aspirates. For cytogenetic analyses $\geq 20$ metaphases were required. AML were classified as cytogenetically normal according to the guidelines of the international system of cytogenetic nomenclature (ISCN) [22]. Mutations of NPM1 [23], FLT3-ITD [24,25], FLT3-TKD [26], moCEBPA and biCEBPA $[6,10]$ and $M L L-P T D ~[27]$ were analyzed as previously published.

\section{Statistical analysis}

The outcome parameter overall survival (OS) was calculated from the date of first diagnosis to death. Relapsefree-survival (RFS) was assessed in all patients having achieved a complete remission (CR) or a CR with incomplete recovery $(\mathrm{CRi})$ according to the standard guidelines of the ELN [28] and was calculated from the date of CR/CRi until relapse or death. For patients who underwent allogeneic SCT OS and RFS times were censored at the date of allogeneic transplantation.

For pairwise comparisons of dichotomous parameters between moCEBPA and biCEBPA-mutated patients the $\chi^{2}$-test/Fisher's exact test was applied. The Mann-Whitney $U$ test was performed for the comparison of continuous parameters between moCEBPA and biCEBPA-mutated patients.

Comparisons of OS and RFS between patients with $\operatorname{moCEBPA}$ and biCEBPA mutations were obtained applying the Kaplan Meier method and the log rank test. Median follow-up was calculated with the reversed Kaplan Meier method. Univariable and multivariable Cox regression analyses were performed for OS and RFS to adjust for potential imbalances of known clinical and molecular prognostic factors summarized in the PINA [29]. To evaluate the effect of biCEBPA vs. moCEBPA mutations on AML-specific survival taking into account only deaths related to AML, a competing risk analysis was performed treating death unrelated to AML, and allogeneic SCT as competing events. Likewise, the cumulative incidence of relapse (CIR) was calculated for all patients in CR/CRi treating death in $\mathrm{CR}$ and allogeneic SCT in first CR as competing events. Cumulative incidence rates [30] and hazard ratios (HR) [31] between the risk groups were calculated and compared by the Gray test [32]. The comparison of OS in biCEBPA- vs. moCEBPA-mutated patients was tested with a significance level of $5 \%$. All other pvalues are descriptive. Analyses were performed using SPSS 
software, version 20.0 (SPSS, Chicago, IL) and the R 3.0.1 software package ( $\mathrm{R}$ foundation for statistical computing, Vienna, Austria).

\section{Results}

Comparison of moCEBPA- vs. biCEBPA-mutated patients with respect to clinical and molecular parameters

Analyses were performed in 88 patients with $\mathrm{CN}-\mathrm{AML}$ and a mutation of CEBPA. Median age was 61 years, the majority (86\%) had de novo AML and an ECOG performance status of 0-2 (96\%). 45 patients showed a biCEBPA mutation, 43 patients had a moCEBPA mutation. Mutations of NPM1 and FLT3-TKD were present in 19\% and 6\%, FLT3-ITD and MLL-PTD occurred in $25 \%$ and $1 \%$ of patients (Additional file 1: Table S1). 67\% of patients received a double induction therapy (Table 1). Median follow-up time was 9.8 years (95\% CI: 9.4-10.1). $4.5 \%$ of patients died within 30 days after start of therapy. Median OS was 3.0 years (95\% CI: 0.9-5.2) and median RFS in 67 patients who have achieved a CR/CRi was 2.3 years (95\% CI: 1.0-3.7) (Table 1). 28 (42\%) of patients in CR/CRi relapsed and 51 (58\%) of patients died.

There were less female patients in the biCEBPA group compared to the moCEBPA group (44\% vs. $72 \%$ ) (Additional file 1: Table S1). The presence of biCEBPA mutations was associated with a higher rate of de novo AML, a higher hemoglobin level, lower platelet count and lower frequencies of additional mutations of NPM1, FLT3-TKD or the presence of FLT3-ITD. All other clinical and molecular parameters as well as therapeutic regimen including allogeneic SCT were evenly distributed between patients with moCEBPA and biCEBPA mutations (Table 1).

\section{biCEBPA-mutated patients show a longer OS, RFS and a lower CIR}

Early death rates at day 30 and at day 60 were not different between patients with biCEBPA and moCEBPA mutations (Table 1).

In accordance with previous results, patients with biCEBPA mutations survived significantly longer compared to those with a moCEBPA mutations (median OS 9.6 years vs. 1.7 years, $\mathrm{p}=0.008$ ) (Figure $1 \mathrm{~A}$ ). This survival benefit was also evident in patients $>60$ years (biCEBPA vs. moCEBPA: 5 -years OS: $37 \%$ vs. $11 \%$, 10 -years OS: $20 \%$ vs. $5 \%, \mathrm{p}=0.045$; (Figure 2C)) and by trend in patients $\leq$ 60 years (biCEBPA vs. moCEBPA: 5-years OS and 10years OS: $81 \%$ vs. $59 \%, \mathrm{p}=0.076$ (Figure $2 \mathrm{~A}$ )).

Consistent with the long follow-up, nine patients died in $\mathrm{CR}$ of causes unrelated to AML. Results of competing risk analyses treating death related to AML and death unrelated to AML as competing are illustrated in the supplement (Additional file 1: Figure S1A). biCEBPA-mutated patients displayed a lower cumulative incidence of death related to AML compared to moCEBPA-mutated patients $(\mathrm{p}=0.028)$ (Additional file 1: Figure S1B).

37 patients (82\%) with biCEBPA mutations and 30 patients $(70 \%)$ with moCEBPA mutations achieved a CR/CRi

Table 1 Therapy and outcome

\begin{tabular}{|c|c|c|c|c|c|c|c|}
\hline & \multicolumn{2}{|c|}{ All patients $(\mathrm{N}=88)$} & \multicolumn{2}{|c|}{ moCEBPA $(\mathrm{N}=43)$} & \multicolumn{2}{|c|}{ biCEBPA $(\mathrm{N}=45)$} & \multirow[t]{2}{*}{$P$} \\
\hline & $\mathrm{N}$ & $\overline{\%}$ & $\bar{N}$ & $\overline{\%}$ & $\bar{N}$ & $\overline{\%}$ & \\
\hline \multicolumn{8}{|l|}{ Number of induction cycles } \\
\hline 1 & 29 & 33 & 16 & 37 & 13 & 30 & 0.41 \\
\hline 2 & 59 & 67 & 27 & 63 & 32 & 71 & \\
\hline Induction therapy regimen & & & & & & & 0.48 \\
\hline In study & 76 & 86 & 36 & 84 & 40 & 89 & \\
\hline In analogy to study & 12 & 14 & 7 & 16 & 5 & 11 & \\
\hline Allogeneic SCT & 19 & 22 & 10 & 23 & 9 & 20 & 0.75 \\
\hline in $1 . C R$ & 7 & 8 & 3 & 7 & 4 & 9 & \\
\hline Primary refractory & 4 & 5 & 3 & 7 & 1 & 2 & \\
\hline At relapse & 8 & 9 & 4 & 9 & 4 & 9 & \\
\hline Early death until day 30 & 4 & 5 & 3 & 7 & 1 & 2 & 0.28 \\
\hline Early death until day 60 & 7 & 8 & 4 & 9 & 3 & 7 & 0.65 \\
\hline $\mathrm{CR} / \mathrm{CRi}{ }^{*}$ & 67 & 76 & 30 & 70 & 37 & 82 & 0.17 \\
\hline Median OS, $95 \% \mathrm{Cl}$, years & \multicolumn{2}{|c|}{$3.0(0.9-5.2)$} & \multicolumn{2}{|c|}{$1.7(0.7-2.7)$} & \multicolumn{2}{|c|}{9.6 (NA) } & 0.008 \\
\hline Median RFS, $95 \% \mathrm{Cl}$, years & \multicolumn{2}{|c|}{$2.3(1.0-3.7)$} & \multicolumn{2}{|c|}{$1.5(0.4-2.5)$} & \multicolumn{2}{|c|}{$9.4(\mathrm{NA})$} & 0.021 \\
\hline
\end{tabular}

*62 patients have achieved a CR; 5 patients have achieved a CRi;

Abbreviations: biCEBPA biallelic mutation in the CCAAT/enhancer-binding protein alpha, $C R$ complete remission, CRi CR with incomplete revovery, moCEBPA monoallelic mutation in the CCAAT/enhancer-binding protein alpha, $N$ number, NA not applicable, SCT stem cell transplantation. 


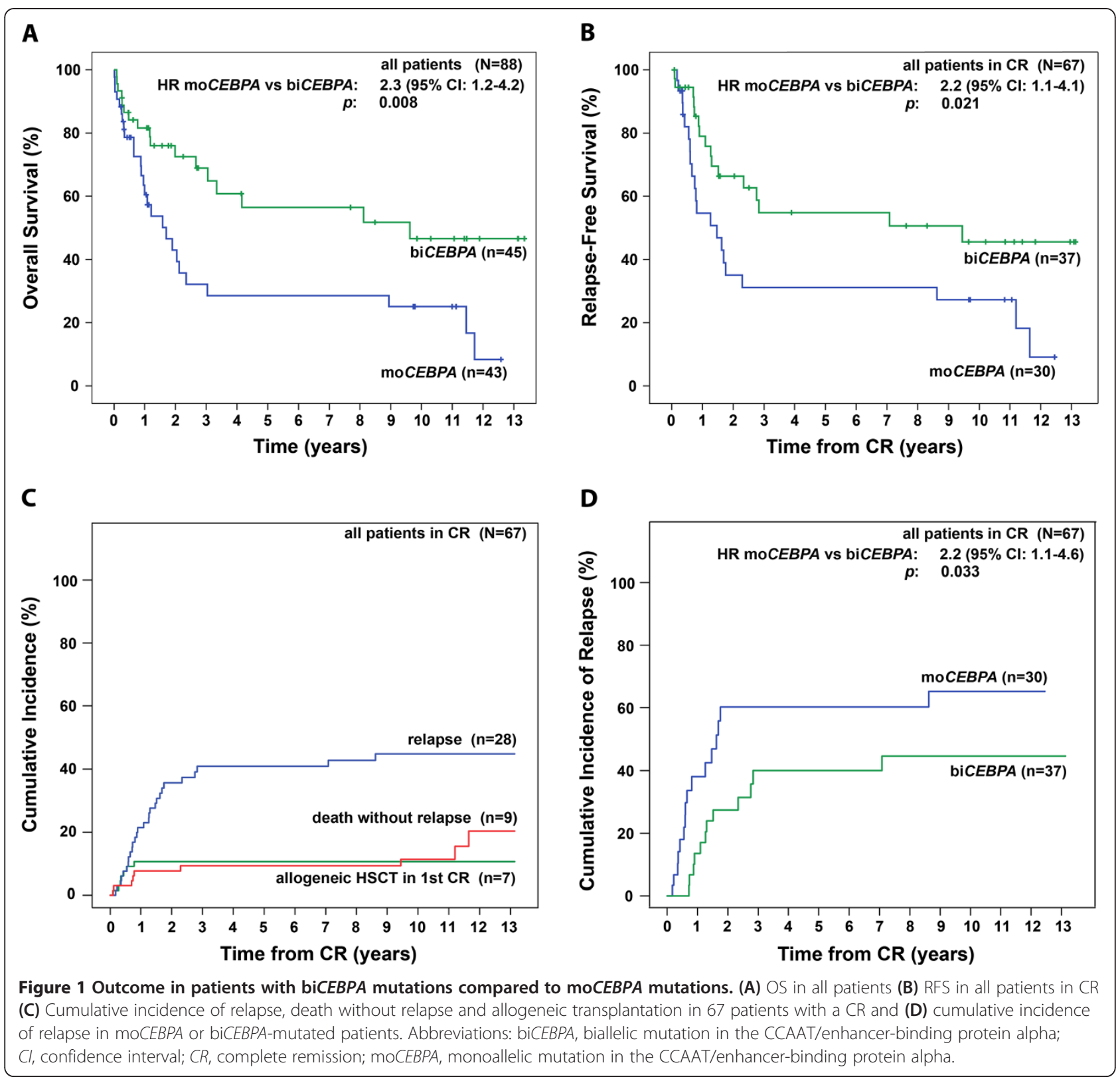

$(\mathrm{p}=0.17)($ Table 1$)$. biCEBPA-mutated patients displayed a longer RFS compared to moCEBPA-mutated patients (median RFS 9.4 years vs. 1.5 years, $\mathrm{p}=0.021$ ) (Figure $1 \mathrm{~B}$, Table 1). A trend to a longer RFS in biCEBPA-mutated patients was seen in subgroups $\leq 60$ and $>60$ years of age ( $\mathrm{p}=0.116$ and $\mathrm{p}=0.082$, respectively) (Figure $2 \mathrm{~B}$ and $\mathrm{D}$ ).

CIR rates were lower in patients carrying biCEBPA compared to moCEBPA mutations (5-year CIR rates: $40 \%$ vs. $60 \%$, respectively; 10 -year CIR rates: 45 vs. $65 \%$, respectively; $\mathrm{p}=0.036$ ) (Figure $1 \mathrm{C}, \mathrm{D})$. Median time from $\mathrm{CR}$ to relapse was longer in the biCEBPA-mutated cohort vs. the moCEBPA-mutated patients (not reached vs. 1.6 years, $\mathrm{p}=0.033)$. In the moCEBPA-mutated patients, $63 \%$ relapsed within the first and 94\% within two years. In contrast, biCEBPA-mutated patients appeared to relapse later during follow-up: only 33\% relapsed in the first year, $67 \%$ within the second year and 92\% within the first three years. In both cohorts, the majority of patient relapsed in the first three years. Two patients - one with a biCEBPA and one with a moCEBPA mutation - showed late relapses after 7 and 8 years. In both cases, we do not have diagnostic bone marrow aspirates to verify if these patients show the same cytogenetic and mutational profile as at diagnosis. Due to the latency of many years and the preceding chemo- and/or radiation therapy, we suspect that these AML relapses might be therapy-associated AML. 


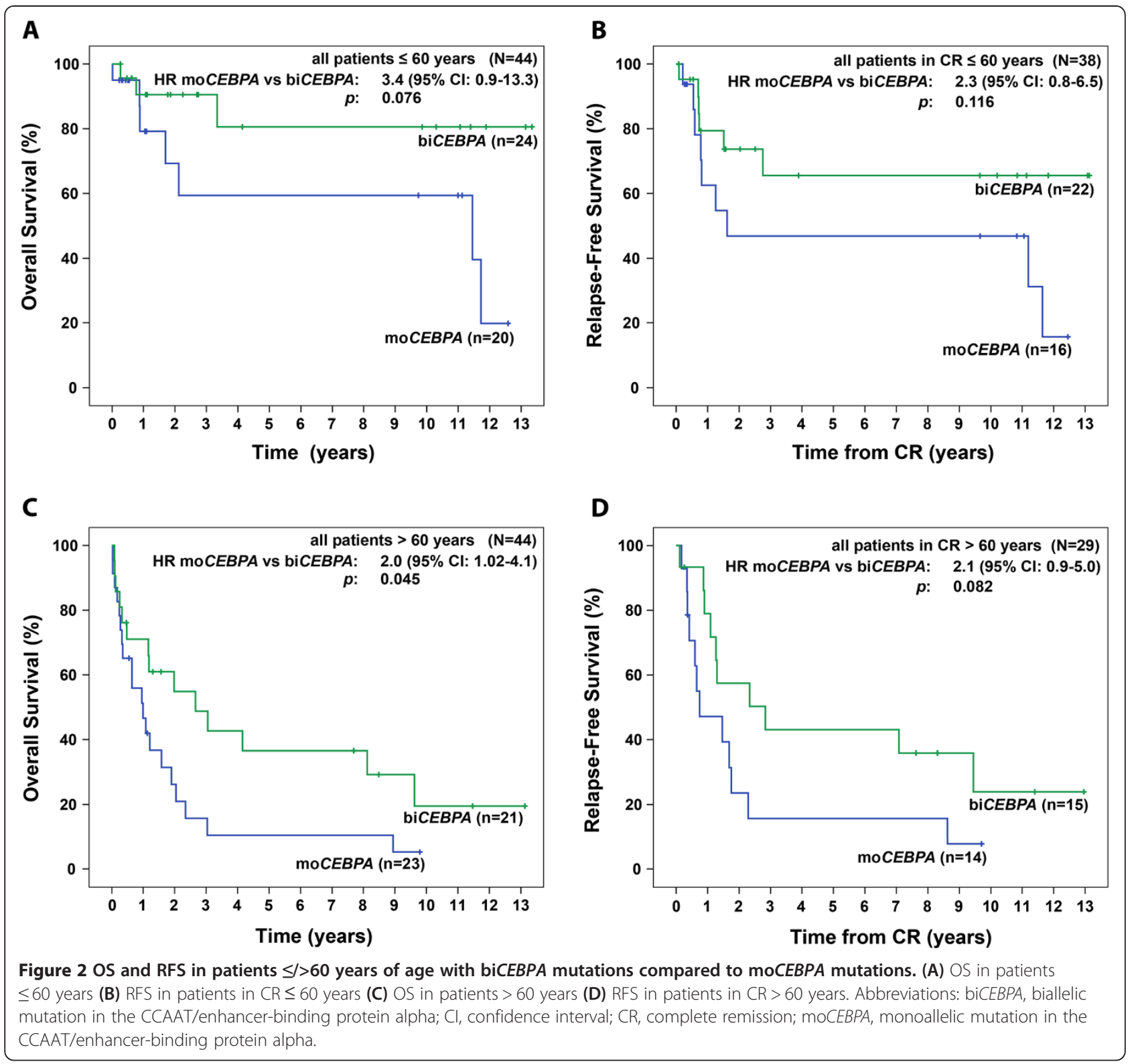

A detailed description of relapse and survival after achievement of a CR according to therapy has been provided in the Additional file 1.

\section{Adjustment for co-occurring mutations and clinical prognostic factors}

Since FLT3-ITD and FLT3-TKD and NPM1 mutations, were more common in moCEBPA-mutated patients, we performed multivariable analyses to adjust for potential confounding effects. The positive impact of biCEBPA vs. moCEBPA mutations on outcome was confirmed when adjusting for co-occurring FLT3-ITD and FLT3-TKD mutations, NPM1 mutations, the PINA $_{\mathrm{OS}}$ and PINA $\mathrm{RFS}_{\mathrm{R}}$ [29] scores without biCEBPA mutations (Table 2) and in analyses with FLT3-wildtype patients only (Additional file 1: Figure $\mathrm{S} 2$ ).

Relapsed patients with biCEBPA and moCEBPA mutations show similar outcomes

Treatment modalities at relapses (palliative vs. intensive treatment; allogeneic SCT) were not different between moCEBPA- and biCEBPA-mutated patients. Both CEBPA cohorts, included similar amounts of patients $\leq 60$ years (moCEBPA: 6/11; biCEBPA 4/9) and > 60 years (moCEBPA: 5/11; biCEBPA 5/9). Although, intensively treated biCEBPAmutated patients showed a tendency to a higher second $C R$ rate compared to moCEBPA-mutated patients ( $78 \%$ vs. $45 \%$, $\mathrm{p}=0.142)$ survival after relapse was not different between patients with moCEBPA or biCEBPA mutations (data not 
Table 2 Cox Regression adjusted for additional markers

\begin{tabular}{|c|c|c|c|c|c|c|}
\hline & \multicolumn{3}{|l|}{ OS } & \multicolumn{3}{|c|}{ RFS } \\
\hline & HR & $95 \% \mathrm{Cl}$ & $\mathrm{p}$ & HR & $95 \% \mathrm{Cl}$ & p \\
\hline biCEBPA versus moCEBPA (univariable analysis) & 0.4 & $0.2-0.8$ & 0.008 & 0.5 & $0.2-0.9$ & 0.021 \\
\hline biCEBPA versus moCEBPA (adjusted for FLT3-ITD) & 0.4 & $0.2-0.8$ & 0.006 & 0.5 & $0.2-0.9$ & 0.021 \\
\hline biCEBPA versus moCEBPA (adjusted for FLT3-TKD) & 0.4 & $0.2-0.8$ & 0.012 & 0.4 & $0.2-0.9$ & 0.018 \\
\hline biCEBPA versus moCEBPA (adjusted for FLT3-ITD and FLT3-TKD) & 0.4 & $0.2-0.8$ & 0.006 & 0.4 & $0.2-0.8$ & 0.012 \\
\hline biCEBPA versus moCEBPA (adjusted for FLT3-ITD, NPM1 mutations and interaction NPM1/FLT3-ITD) & 0.2 & $0.1-0.5$ & $<0.001$ & 0.2 & $0.1-0.6$ & 0.003 \\
\hline biCEBPA versus moCEBPA (adjusted for NPM1 mutations) & 0.2 & $0.1-0.5$ & $<0.001$ & 0.2 & $0.1-0.6$ & 0.002 \\
\hline 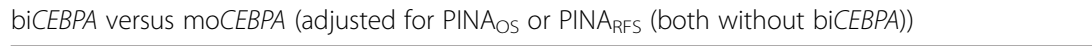 & 0.2 & $0.1-0.4$ & $<0.001$ & 0.2 & $0.1-0.6$ & 0.001 \\
\hline
\end{tabular}

Abbreviations: biCEBPA biallelic mutation in the CCAAT/enhancer-binding protein alpha, Cl confidence interval, FLT3-ITD internal tandem duplication of the FLT3 gene, FLT3-TKD mutation in the tyrosine kinase domain of the FLT3 gene, HR hazard ratio, interaction NPM1/FLT3-ITD, NPM1 positive/FLT3-ITD positive versus NPM1 negative or FLT3-ITD negative, moCEBPA monoallelic mutation in the CCAAT/enhancer-binding protein alpha, NPM1, nucleophosmin gene, OS Overall survival, $p$ p value, $P I N A_{O S}$ Prognostic Index for OS in cytogenetically normal AML [29], PINA $A_{R F S}$ Prognostic Index for RFS in cytogenetically normal AML [29], RFS Relapse-free survival.

shown). In both cohorts, long-term survival after relapse was only possible for patients treated with allogeneic SCT. A detailed description of treatment at relapse, achievement of a second CR and survival is given in the Additional file 1 .

\section{Discussion}

According to the current WHO classification and the ELN guidelines patients with mutated CEBPA represent a cohort with a favorable prognosis $[1,28]$. We and others have shown before, that this favorable prognostic effect is restricted to the group of patients with biCEBPA, in contrast to moCEBPA [6] mutations. The aim of our current analysis was to test if the favorable prognostic effect of biCEBPA mutations was still evident within a (1) larger patient cohort and (2) after a longer follow-up period.

Due to the low frequency of $C E B P A$ mutations of about $8-18 \%$ in CN-AML, studies are often based on small patient numbers $[6,13,15]$, which reduces statistical power. Our study includes 88 patients, 75 of whom were treated homogeneously within AMLCG99 and AMLCG2008 trials.

Except for the study of Green et al. [14] with a medium follow-up for survivors of 11.7 years, median follow-up in the published literature including our previous publication is mostly $\leq 5$ years $[6,13,15,16,33]$. The present analysis has a long medium follow-up of almost 10 years (9.8 years) and a maximum follow-up of 13.3 years. This allowed to detect late relapses.

In accordance with previous analyses [13-15], biCEBPAmutated AML patients displayed a significantly longer OS compared to moCEBPA-mutated patients. Differences in OS might be caused by differences in early death rate, achievement of a CR and RFS/incidence of relapses. Patients with a biCEBPA mutation showed a slightly lower early death rate at day 60 (6.7\% versus 9.3\%, Table 1 ) and a higher $C R$ rate (CR rates: $82 \%$ versus $70 \%, \mathrm{p}=0.17$, respectively) compared to patients with a moCEBPA mutation. Interestingly, overall early death rate until day 60 in all CEBPA-mutated patients in our cohort was $8.0 \%$, which is lower compared to data in the literature of $10 \%$ to $16 \%[19,20,34]$. These differences might be caused by the fact that these studies included all cytogenetic risk groups and not only CN-AML patients. Most importantly, we could clearly see a better RFS in biCEBPA-mutated patients. This improved RFS was also seen by others [14,15]. Moreover, we could demonstrate a lower CIR in biCEBPA-mutated compared to moCEBPA-mutated patients. However, to what extent a lower early death rate, a higher $\mathrm{CR}$ rate or a longer RFS caused the prolonged survival still remains open and needs to be investigated in larger patient cohorts.

The favorable prognosis of biCEBPA-mutated AML was most evident in patients $\leq 60$ years who displayed a 10 -years OS of $81 \%$ and a 10 -years RFS of $66 \%$.

In contrast to relapse in moCEBPA-mutated patients, occurring in the majority of patients $(63 \%)$ within the first year after achievement of a CR, only about one third of biCEBPA-mutated patients relapsed within the first year. Almost all moCEBPA-mutated patients and biCEBPA-mutated patients relapsed within the first three years.

Interestingly, one patient with a biCEBPA-mutated AML and one patient with a moCEBPA-mutated AML relapsed more than 7 years after $C R$. To our knowledge, this is the first case of such a late relapse in biCEBPA-mutated AML. These late AML relapses might be therapy-associated and display different cytogenetics and molecular mutational patterns. Unfortunately, due to lack of material, we cannot confirm the presence of a CEBPA mutation at relapse. Both cases underline the importance of a long-term follow-up of patients with AML and CEBPA mutations.

The long patient follow-up enabled us to detect patients with late deaths ( $>5$ years) unrelated to AML: Two patients with a moCEBPA mutation and one patient with a biCEBPA mutation died in CR after 11.7 years (cause unknown), 11.5 years (cause: development of a cancer of 
unknown primary) and 9.6 years (cause: cardiac failure). Thus, the inclusion of "AML unrelated death" helps to better estimate prognosis, especially in elderly co-morbide patients.

Schlenk et al. [35] recently showed in a large cohort of 124 CN-AML patients with biCEBPA mutations in first $\mathrm{CR}$, a significantly longer RFS, but similar OS, for those patients receiving allogeneic or autologous transplantation compared to chemotherapy only. We also found a similar OS in our biCEBPA-mutated patients $(\mathrm{n}=4)$ receiving allogeneic $\mathrm{SCT}$ in first $\mathrm{CR}$, compared to those obtaining chemotherapy $(n=30)$, although we could not detect an effect of allogeneic SCT on RFS, which might be due to the small number of patients receiving allogeneic SCT (data not shown). In accordance with Schlenk et al. [35] we found a high second CR rate after reinduction therapy of $78 \%$ in biCEBPA-mutated patients treated with intensive protocols. Due to transplant-related mortality, infections and a high relapse rate after the second $\mathrm{CR}$, this did not result in a longer OS compared to relapsed moCEBPA-mutated patients. These results have to be interpreted with caution since they are limited by small patient numbers. Schlenk et al. [35] furthermore showed that only relapsed patients treated with allogeneic SCT, but not those treated with chemotherapy alone, survived longer than 2 years. Our analyses - although performed in a smaller patient cohort - are in line with these results: In our cohort, patients with biCEBPA mutations that have received allogeneic SCT at the time of relapse are still alive (after 7.0 and 11.3 years) or have died due to treatment related mortality, but not due to AML relapse/refractory AML. In contrast, all 5 patients receiving chemotherapy at the time of relapse died (median OS after relapse: 0.9 years).

\section{Conclusion}

In conclusion, our study with a long-term follow-up of homogeneously treated CN-AML of almost 10 years clearly showed that patients carrying biCEBPA mutations have a substantially better OS and RFS as well as a relevantly lower CIR compared to patients with moCEBPA mutations. The excellent prognosis of younger AML patients with biCEBPA mutations (10-year OS of $81 \%$ ) might lead to a reduction of the intensity of postremission therapy in this subgroup.

\section{Consent}

Written informed consent was obtained from patients for the data collection, analysis and publication.

\section{Additional file}

Additional file 1: Data supplement.

\section{Competing interests}

The authors indicate no potential conflicts of interest.

\section{Authors' contributions}

Conception and design: FP, DK, WH, KS. Collection and assembly of data: FP, DK, AD, NPK, SS, JB, MCS, WEB, TB, BW, WH, KS. Statistical review: EH. Data analysis and interpretation: FP, DK, WH, KS. Manuscript writing: All authors. All authors read and approved the final manuscript.

\section{Acknowledgement}

We would like to thank all centers and patients that participated in this study and Gudrun Mellert, Evelyn Zellmeier and Bianka Ksienzyk for their excellent technical assistance.

\section{Funding}

Supported by Deutsche Forschungsgemeinschaft Grant SFB 684/A 12 (K.S) and by the German Cancer Consortium (DKTK) and the German Cancer Research Center (DKFZ), Heidelberg, Germany.

\section{Author details}

${ }^{1}$ Laboratory for Leukemia Diagnostics, Department of Internal Medicine III, University Hospital Munich Grosshadern, Munich, Germany. ${ }^{2}$ German Cancer Consortium (DKTK), Heidelberg, Germany. ${ }^{3}$ German Cancer Research Center (DKFZ), Heidelberg, Germany. ${ }^{4}$ Department of Medical Statistics, Biometry and Epidemiology, University Munich, Munich, Germany. ${ }^{5}$ Institute of Biostatistics and Clinical Research, University of Muenster Germany, Muenster, Germany. ${ }^{6}$ Department of Medicine A, Hematology and Oncology, University of Muenster, Muenster, Germany. ${ }^{7}$ German Society of Hematology and Oncology, Berlin, Germany. ${ }^{8}$ Department of Oncology and Hematology, Klinikum Barmherzige Brüder, Regensburg, Germany.

Received: 22 May 2014 Accepted: 24 July 2014

Published online: 10 September 2014

\section{References}

1. Swerdlow SH, International Agency for Research on Cancer: WHO Classification of Tumours of Haematopoietic and Lymphoid Tissues. World Health Organization; 2008.

2. Swart GW, van Groningen JJ, van Ruissen F, Bergers M, Schalkwijk J: Transcription factor C/EBPalpha: novel sites of expression and cloning of the human gene. Biol Chem 1997, 378:373-379.

3. Pabst T, Mueller BU, Zhang P, Radomska HS, Narravula S, Schnittger S, Behre G, Hiddemann W, Tenen DG: Dominant-negative mutations of CEBPA, encoding CCAAT/enhancer binding protein-[alpha] (C/EBP[alpha]), in acute myeloid leukemia - Nature Genetics. Nat Genet 2001, 27:263-270.

4. Pabst T, Mueller BU: Transcriptional dysregulation during myeloid transformation in AML. Oncogene 2007, 26:6829-6837.

5. Zhang DE, Zhang P, Wang ND, Hetherington CJ, Darlington GJ, Tenen DG: Absence of granulocyte colony-stimulating factor signaling and neutrophil development in CCAAT enhancer binding protein alphadeficient mice. Proc Natl Acad Sci U S A 1997, 94:569-574.

6. Dufour A, Schneider F, Metzeler KH, Hoster E, Schneider S, Zellmeier E, Benthaus T, Sauerland M-C, Berdel WE, Büchner T, Wörmann B, Braess J, Hiddemann W, Bohlander SK, Spiekermann K: Acute myeloid leukemia with biallelic CEBPA gene mutations and normal karyotype represents a distinct genetic entity associated with a favorable clinical outcome. J Clin Oncol 2010, 28:570-577.

7. Fröhling S, Schlenk RF, Stolze I, Bihlmayr J, Benner A, Kreitmeier S, Tobis K, Dohner $H$, Döhner K: CEBPA mutations in younger adults with acute myeloid leukemia and normal cytogenetics: prognostic relevance and analysis of cooperating mutations. J Clin Oncol 2004, 22:624-633.

8. Bienz M, Ludwig M, Leibundgut EO, Mueller BU, Ratschiller D, Solenthaler M, Fey MF, Pabst T: Risk assessment in patients with acute myeloid leukemia and a normal karyotype. Clin Cancer Res 2005, 11:1416-1424.

9. Schlenk RF, Döhner K, Krauter J, Fröhling S, Corbacioglu A, Bullinger L, Habdank M, Späth D, Morgan M, Benner A, Schlegelberger B, Heil G, Ganser A, Dohner H, German-Austrian Acute Myeloid Leukemia Study Group: Mutations and treatment outcome in cytogenetically normal acute myeloid leukemia. N Engl J Med 2008, 358:1909-1918.

10. Benthaus T, Schneider F, Mellert G, Zellmeier E, Schneider S, Kakadia PM, Hiddemann W, Bohlander SK, Feuring-Buske M, Braess J, Spiekermann K, 
Dufour A: Rapid and sensitive screening for CEBPA mutations in acute myeloid leukaemia. Br J Haematol 2008, 143:230-239.

11. Snaddon J, Smith ML, Neat M, Cambal-Parrales M, Dixon-Mclver A, Arch R, Amess JA, Rohatiner AZ, Lister TA, Fitzgibbon J: Mutations of CEBPA in acute myeloid leukemia FAB types M1 and M2. Genes Chromosom Cancer 2003, 37:72-78

12. Van Doorn S, Erpelinck C: Biallelic mutations in the CEBPA gene and low CEBPA expression levels as prognostic markers in intermediate-risk AML. Hematol J 2003, 4:31-40.

13. Wouters BJ, Lowenberg B, Erpelinck-Verschueren CAJ, Van Putten WL, Valk PJM, Delwel R: Double CEBPA mutations, but not single CEBPA mutations, define a subgroup of acute myeloid leukemia with a distinctive gene expression profile that is uniquely associated with a favorable outcome. Blood 2009, 113:3088-3091.

14. Green CL, Koo KK, Hills RK, Burnett AK, Linch DC, Gale RE: Prognostic significance of CEBPA mutations in a large cohort of younger adult patients with acute myeloid leukemia: impact of double CEBPA mutations and the interaction with FLT3 and NPM1 mutations. J Clin Oncol 2010, 28:2739-2747.

15. Pabst T, Eyholzer M, Fos J, Mueller BU: Heterogeneity within AML with CEBPA mutations; only CEBPA double mutations, but not single CEBPA mutations are associated with favourable prognosis. Br J Cancer 2009, 100:1343-1346.

16. Taskesen E, Bullinger L, Corbacioglu A, Sanders MA, Erpelinck CAJ, Wouters BJ, van der Poel-van de Luytgaarde SC, Damm F, Krauter J, Ganser A, Schlenk RF, Löwenberg B, Delwel R, Dohner H, Valk PJM, Döhner K: Prognostic impact, concurrent genetic mutations, and gene expression features of AML with CEBPA mutations in a cohort of 1182 cytogenetically normal AML patients: further evidence for CEBPA double mutant AML as a distinctive disease entity. Blood 2011, 117:2469-2475.

17. Dufour A, Schneider F, Hoster E, Benthaus T, Ksienzyk B, Schneider S, Kakadia PM, Sauerland M-C, Berdel WE, Büchner T, Wörmann B, Braess J, Subklewe M, Hiddemann W, Bohlander SK, Spiekermann K, AML CG study group: Monoallelic CEBPA mutations in normal karyotype acute myeloid leukemia: independent favorable prognostic factor within NPM1 mutated patients. Ann Hematol 2012, 91:1051-1063.

18. Oken MM, Creech RH, Tormey DC, Horton J, Davis TE, McFadden ET, Carbone PP: Toxicity and response criteria of the Eastern Cooperative Oncology Group. Am J Clin Oncol 1982, 5:649-655.

19. Büchner T, Berdel WE, Schoch $C$, Haferlach T, Serve HL, Kienast J, Schnittger S, Kern W, Tchinda J, Reichle A, Lengfelder E, Staib P, Ludwig W-D, Aul C, Eimermacher H, Balleisen L, Sauerland M-C, Heinecke A, Wörmann B, Hiddemann W: Double induction containing either two courses or one course of high-dose cytarabine plus mitoxantrone and postremission therapy by either autologous stem-cell transplantation or by prolonged maintenance for acute myeloid leukemia. J Clin Oncol 2006, 24:2480-2489.

20. Braess J, Spiekermann K, Staib P, Grüneisen A, Wörmann B, Ludwig W-D, Serve H, Reichle A, Peceny R, Oruzio D, Schmid C, Schiel X, Hentrich M, Sauerland C, Unterhalt M, Fiegl M, Kern W, Buske C, Bohlander S, Heinecke A, Baurmann H, Beelen DW, Berdel WE, Büchner T, Hiddemann W: Dose-dense induction with sequential high-dose cytarabine and mitoxantone (S-HAM) and pegfilgrastim results in a high efficacy and a short duration of critical neutropenia in de novo acute myeloid leukemia: a pilot study of the AMLCG. Blood 2009, 113:3903-3910.

21. Tobis K, Schlenk RF, Liebisch C, Frohling S, Döhner H, Dohner K: Identification of partial tandem duplications of the MLL gene in acute myeloid leukemia: prospective analysis within the multicenter treatment trial AML HD98-a. Acute Leukemias IX Hematology Blood Transfusion 2003, 41:306-311.

22. Shaffer LG, McGowan-Jordan J, Schmid M: ISCN 2013: An International System for Human Cytogenetic Nomenclature (2013). Recommendations of the International Standing Committee on Human Cytogenetic Nomenclature. In Basel, Switzerland: Karger publishers; 2013.

23. Falini B, Mecucci C, Tiacci E, Alcalay M, Rosati R, Pasqualucci L, La Starza R, Diverio D, Colombo E, Santucci A, Bigerna B, Pacini R, Pucciarini A, Liso A, Vignetti M, Fazi P, Meani N, Pettirossi V, Saglio G, Mandelli F, Coco Lo F, Pelicci P-G, Martelli MF, GIMEMA Acute Leukemia Working Party: Cytoplasmic nucleophosmin in acute myelogenous leukemia with a normal karyotype. N Engl J Med 2005, 352:254-266.

24. Fröhling S, Schlenk RF, Breitruck J, Benner A, Kreitmeier S, Tobis K, Dohner H, Döhner K, AML Study Group UIm. Acute myeloid leukemia: Prognostic significance of activating FLT3 mutations in younger adults (16 to 60 years) with acute myeloid leukemia and normal cytogenetics: a study of the AML Study Group Ulm. Blood 2002, 100:4372-4380

25. Thiede C, Steudel C, Mohr B, Schaich M, Schäkel U, Platzbecker U, Wermke M, Bornhäuser M, Ritter M, Neubauer A, Ehninger G, Illmer T: Analysis of FLT3-activating mutations in 979 patients with acute myelogenous leukemia: association with $\mathrm{FAB}$ subtypes and identification of subgroups with poor prognosis. Blood 2002, 99:4326-4335.

26. Bacher U, Haferlach C, Kern W, Haferlach T, Schnittger S: Prognostic relevance of FLT3-TKD mutations in AML: the combination matters-an analysis of 3082 patients. Blood 2008, 111:2527-2537.

27. Schnittger S, Kinkelin U, Schoch C, Heinecke A, Haase D, Haferlach T, Büchner T, Wormann B, Hiddemann W, Griesinger F: Screening for MLL tandem duplication in 387 unselected patients with AML identify a prognostically unfavorable subset of AML. Leukemia 2000, 14:796-804

28. Döhner H, Estey EH, Amadori S, Appelbaum FR, Büchner T, Burnett AK, Dombret $H$, Fenaux P, Grimwade D, Larson RA, Coco Lo F, Naoe T, Niederwieser D, Ossenkoppele GJ, Sanz MA, Sierra J, Tallman MS Löwenberg B, Bloomfield CD, European LeukemiaNet: Diagnosis and management of acute myeloid leukemia in adults: recommendations from an international expert panel, on behalf of the European LeukemiaNet. Blood 2010, 115:453-474.

29. Pastore F, Dufour A, Benthaus T, Metzeler KH, Maharry KS, Schneider S, Ksienzyk B, Mellert G, Zellmeier E, Kakadia PM, Unterhalt M, Feuring-Buske M, Buske C, Braess J, Sauerland M-C, Heinecke A, Krug U, Berdel WE, Buechner T, Woermann B, Hiddemann W, Bohlander SK, Marcucci G, Spiekermann K, Bloomfield CD, Hoster E: Combined molecular and clinical prognostic index for relapse and survival in cytogenetically normal acute myeloid leukemia. J Clin Oncol 2014, 32:1586-1594.

30. Kalbfleisch JD, Prentice RL: The Statistical Analysis of Failure Time Data. In New York: John Wiley; 1980.

31. Fine JP, Gray RJ: A proportional hazards model for the subdistribution of a competing risk. J Am Stat Assoc 1999, 94:496-509.

32. Gray RJ: A class of K-sample tests for comparing the cumulative incidence of a competing risk. Ann Stat 1988, 16:1141-1154.

33. Renneville A, Boissel N, Gachard N, Naguib D, Bastard C, Nibourel O, Pautas C, Reman O, Thomas X, Gardin C, Terré C, Castaigne S, Preudhomme C, Dombret $\mathrm{H}$ : The favorable impact of CEBPA mutations in patients with acute myeloid leukemia is only observed in the absence of associated cytogenetic abnormalities and FLT3 internal duplication. Blood 2009, 113:5090-5093.

34. Walter RB, Othus M, Borthakur G, Ravandi F, Cortes JE, Pierce SA Appelbaum FR, Kantarjian HA, Estey EH: Prediction of early death after induction therapy for newly diagnosed acute myeloid leukemia with pretreatment risk scores: a novel paradigm for treatment assignment. J Clin Oncol 2011, 29:4417-4423.

35. Schlenk RF, Taskesen E, van Norden Y, Krauter J, Ganser A, Bullinger $L$, Gaidzik VI, Paschka P, Corbacioglu A, Göhring G, Kündgen A, Held G, Götze K, Vellenga E, Kuball J, Schanz U, Passweg J, Pabst T, Maertens J, Ossenkoppele GJ, Delwel R, Dohner H, Cornelissen JJ, Döhner K, Löwenberg B: The value of allogeneic and autologous hematopoietic stem cell transplantation in prognostically favorable acute myeloid leukemia with double mutant CEBPA. Blood 2013, 122:1576-1582.

doi:10.1186/s13045-014-0055-7

Cite this article as: Pastore et al:: Long-term follow-up of cytogenetically normal CEBPA-mutated AML. Journal of Hematology \& Oncology 2014 7:55. 